\title{
BEAM-GAS LIFETIME IN A LOW EMITTANCE, MEDIUM-ENERGY STORAGE RING*
}

\author{
S. M. Chung, C. D. Park, S. H. Gu, and M. Kwon \\ Pohang Accelerator Laboratory, Pohang University of Science and Technology, \\ Pohang, Kyungbuk 790-784, Korea
}

\begin{abstract}
In order to understand the beam-lifetime limiting process in a medium-energy machine like the $2 \mathrm{GeV}$ Pohang Light Source, the beam-gas scattering effects have been measured by using helium gas and the effects of the transverse aperture height on the lifetime have also been examined by using a beam scraper. It is found that for a medium to large gap vacuum chamber the lifetime is mainly limited by Touschek effect at the machine operating pressure, low $10^{-9}$ Torr. For a very small gap undulator, however, the beam-gas scattering makes substantial contribution to the beam lifetime even at $\sim 1 \times 10^{-9}$ Torr because of Coulomb scattering.
\end{abstract}

\section{INTRODUCTION}

The Pohang Light Source (PLS) of the Pohang Accelerator Laboratory is a low emittance third generation synchrotron radiation source with a nominal electron beam energy of $2 \mathrm{GeV}$. The storage ring vacuum system of the PLS has been designed to maintain the average pressure of 1 nTorr over the $280 \mathrm{~m}$ circumference with a circulating current of $400 \mathrm{~mA}$ to enable beam lifetimes longer than 10 hours.

The performance of the vacuum system is measured primarily in terms of beam lifetime, and achieving a long lifetime is a key issue for the third generation light sources. In PLS, the lifetime of 100-mA beam in the early stage of the commissioning was less than 50 minutes due to a poor vacuum. However, gradual decrease in pressure due to synchrotron radiation cleaning of the vacuum chamber resulted in continuous improvement in lifetime. To date a total beam dose of 1600 Ampere-hour has been accumulated and beam lifetimes in excess of 20 hours with $170 \mathrm{~mA}$ of stored beam are regularly obtained.

As in the case of high-energy machines, probably scattering of beam electrons on residual gas molecules is the most important physical process determining the beam lifetime.[1] Furthermore, the narrow gap undulators foreseen in the straight sections make the machine particularly sensitive to beam-gas scattering.

However, due to the intrinsic small emittance coupling ( $<1 \%$ ), the lifetime may also be limited by pressure independent intra-beam scattering (Touschek effect)[2], which is proportional to the electron density in the bunch. Moreover, increasing the electron density to increase beam current results in enhancing the intra-beam scattering rate and reducing Touschek lifetime. After all, unlike low or high energy machines, the lifetime limiting factor which will prevail in a medium energy machine like the $2 \mathrm{GeV}$ PLS depends on the competition between the intra-beam and beam-gas scattering. The question of which of the two scattering effects dominates the lifetime in the specific pressure then remains open.

In order to help resolve these difficulties, the beam-gas lifetimes were measured by using helium in the pressure range from $\sim 5 \times 10^{-9}$ to $\sim 3 \times 10^{-7}$ Torr. The effect of the accommodation of a small-gap undulator on the lifetime was also examined. The implications of the results of the lifetime measurements will be discussed in terms of the lifetime limiting process in PLS storage ring.

\section{EXPERIMENTS}

Inert gas helium was used for the present work to control the chamber pressure. By introducing helium gas into the storage ring with sputter ion pumps (SIPs) switched off, we can make the pressure uniform all over the circumference, whereas other active gases are continuously pumped by non-evaporable getters. Then the beam-gas lifetime can be easily measured and calculated using the uniform and monitored pressure with average beta function.

Besides its vacuum characteristics, the light mass of helium makes sure that helium gas is cleared by the long bunch gap. And the beam-gas scattering effect for helium is close to that for the residual gas at the operating pressures of a storage ring. Note that the ratio of the total scattering cross section or the beam lifetime for helium, to that for the residual gas measured during the machine operation $\left(90 \% \mathrm{H}_{2}, 2 \% \mathrm{CH}_{4}, 1 \% \mathrm{H}_{2} \mathrm{O}, 6 \% \mathrm{CO}\right.$ and $1 \%$ $\mathrm{CO}_{2}$ ) is $\sim 2.2$. Therefore we can simulate the beam-gas lifetime as close as the real situation.

The beam lifetimes were measured with/without a medium-gap undulator. The base pressure was $\sim 5 \times 10^{-10}$ Torr without beam and was $\sim(1 \sim 4) \times 10^{-9}$ Torr during measurements, depending on the beam current and also on the SIPs on and/or off. The electron beam was stored to a value, which is lower than the threshold current of the strong collective instabilities induced by the high order mode of the RF cavities. Helium gas was admitted step by step into the storage ring with increasing pressures and the helium pressure was stabilised in few minutes. Finally, the 
beam-gas lifetimes for the small gap undulators were simulated using a beam scraper. Since insertion devices for the third generation light sources generally varies with the vertical gap, we measured the lifetime with a verticalbeam scraper only.

\section{RESULTS AND DISCUSSION}

The measured beam lifetimes, $\tau_{\mathrm{He}}$ (Fig. 1(•)), for the PLS storage ring having a medium gap undulator is shown in Fig. 1. The corresponding acceptance limiting halfaperture is $6 \mathrm{~mm}$ (vertical). The calculated beam-gas (Fig. $1(\Delta)$ ), Touschek (Fig. 1(+)) and total lifetimes (Fig. 1, solid line) are also shown for comparison. It is clear from the figure that the measured lifetime for helium depends linearly on the pressure at high helium pressures, $\geq 1 \times 10^{-7}$ Torr (Fig. 1(c)), indicating that the beam-gas scattering effect dominates the total lifetime.

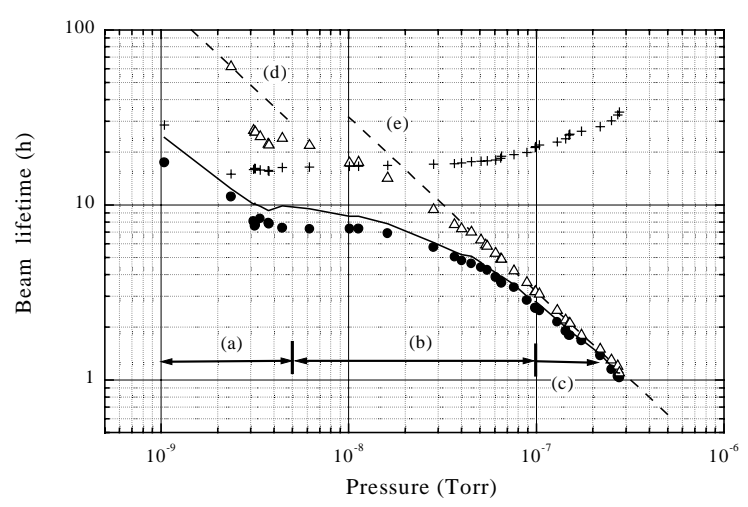

Figure 1: Measured (•) and calculated lifetimes. The initial stored current was $167 \mathrm{~mA}$ in 250 bunches.

In the helium pressure range from $\sim 5 \times 10^{-9}$ to $\sim 1 \times 10^{-7}$ Torr (Fig. 1(b)), it can be stated that the beam-gas scattering effect competes with Touschek effect. At a point in this pressure range, both effects make equal contribution to the total lifetime for the Touschek lifetime changes greatly according to the number of electrons per bunch and the machine coupling while the beam-gas lifetime is constant at a given pressure.

Below the pressure of $\sim 5 \times 10^{-9}$ Torr (Fig. 1(a)), we could not measure the lifetime with helium. As the SIPs were switched off, the pressure went up to $\sim 3.5 \times 10^{-9}$ Torr. And the lifetime was reduced immediately by $\sim 25 \%$ according to the pressure change. The result directly reflects the importance of the beam-gas scattering effect in the low $10^{-}$

${ }^{9}$ Torr range. Nevertheless, we can find from the figure that Touschek effect mainly limits the lifetime in low $10^{-9}$ Torr.

In the meantime, the measured lifetimes in the highpressure ranges $\left(\geq 5 \times 10^{-8}\right.$ Torr) where the helium content was at least $\geq 97 \%$ closely followed the predicted values, meaning that a good knowledge of the machine parameters had been made. With the agreement of the measured and calculated beam lifetimes for helium, the beam-gas lifetimes were studied analytically based on the equations and the residual gas described in section 2 .

The calculations show that with large transverse apertures, Bremsstrauhlung $\left(\tau_{\mathrm{B}} \approx 409\right.$ [nTorr $\bullet$ h] $)$ is dominant beam-gas interaction for the PLS as in most other machines. As the vertical gap is decreased, the Coulomb scattering becomes important. Both the Coulomb and Bremsstrauhlung lifetime are comparable at a gap half-height of $8 \sim 9 \mathrm{~mm}$. With a medium gap undulator $(6 \mathrm{~mm})$, the beam-gas lifetime $\left(\tau_{\text {gas }}\right)$ for the PLS storage ring is $\mathrm{P} \bullet \tau \approx 145$ [nTorr $\bullet$ h] (Fig. 1(d)). In this regard, if we directly compare $\tau_{\text {gas }}$ with $\tau_{\mathrm{He}}$ (Fig. 1(e)), the pressure at which the beam-gas scattering effect dominates may corresponds to $\sim 5 \times 10^{-8}$ Torr. And from $\sim 5 \times 10^{-9}$ Torr to $\sim 5 \times 10^{-8}$ Torr, both effect may competes and finally Touschek effect dominates the lifetime below middle $10^{-9}$ Torr.

On the other hand, for a very small gap undulator to be installed in straight sections, the Coulomb scattering effect will be significant. Since undulators become narrower and narrower, the beam lifetimes were examined with a vertical-beam scraper to see the beam-gas scattering effect in low $10^{-9}$ Torr in detail. Figure 2 gives the beam-gas lifetimes measured as a function of the vertical aperture height at two different operating pressures of $5 \times 10^{-10}$ Torr and $1 \times 10^{-9}$ Torr. The beam current of $\sim 5 \mathrm{~mA}$ was stored in 350 bunches to minimise Touschek effect. The calculated beam lifetimes using the measured residual gas and the machine parameters are also shown in Fig. 2. Discrepancies between the measure and calculated lifetimes reflect the non-uniform pressure distributions, which makes it difficult to fit the measured data to calculated ones.

It can be found that the contribution of the beam-gas scattering effect to the total lifetime is small for a medium gap undulator, 5 6 $\mathrm{mm}$. (Note that the Touschek lifetime $\left(\tau_{\mathrm{C}}\right)$ ranges from a few hours to $\sim 30$ hours). However, for the aperture half-height of, around $3 \sim 4 \mathrm{~mm}$ the beam-gas scattering may compete with the Touschek effect even at low $10^{-9}$ Torr because of Coulomb scattering which varies with the transverse aperture size while Bremsstrahlung keeps constant at a given pressure. The lifetime can be further reduced due to small vertical gap of the insertion devices since the $\tau_{\mathrm{C}}$ depends indirectly on the transverse aperture because of the reduced momentum aperture of the storage ring. Thus the bean-gas scattering effect for a very small gap undulator will be critical factor to make decisions to keep the best machine performance. The measurement also shows that the beam-gas lifetime of the 2-GeV PLS storage ring will be within the shaded area in Fig. 2, depending on the pressure and the vertical gap of the insertion devices. 


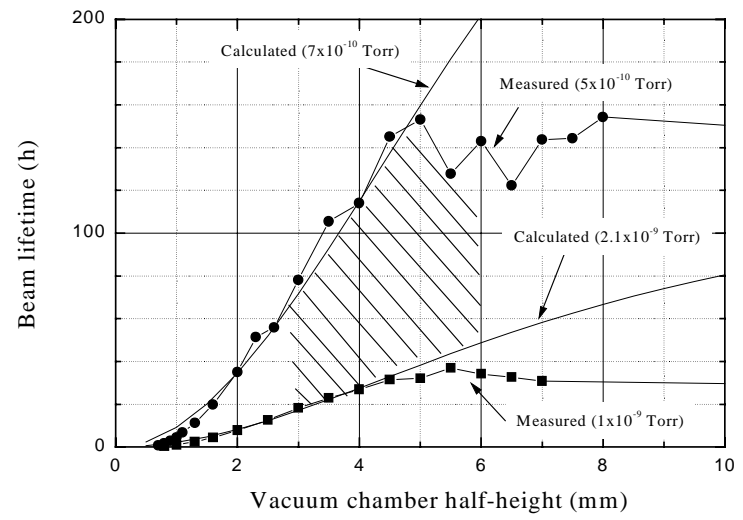

Figure 3. Measured beam-gas lifetimes using a vertical scraper. The solid lines are fitted to the measured data by varying pressure.

In order to compensate for the degradation in the machine performance because of small vertical gap, various methods have been studied in many storage rings regardless of their energy. For example, the longer beam lifetime can be achieved by increasing the momentum acceptance of the storage ring, by manipulating bunch dimensions, by minimising beta function in the insertion straight region, and by using the so-called "In-Vacuum Undulator". [4-5]

A simple method to increase the lifetime is to decrease Touschek effect by reducing the current density in bunches. But in general the brightness must be compromised with the lifetime. Increasing the beam energy to an available amount can be a possible way to increase the beam lifetime without degradation of beam quality. For example, about $30 \%$ increment in beam lifetime with $2.4 \mathrm{GeV}$ already achieved for the PLS (Note that Coulomb and Touschek lifetimes are proportional to $\mathrm{E}^{2}$ and $\mathrm{E}^{3}$, respectively). However we may loose the low energy photons to some extent.

From the vacuum point of view, the lower pressure can compensate for the reduction in lifetime. As high as $\sim 50 \%$ increment in lifetime can be achieved by reducing the pressure from $\sim 2 \times 10^{-9}$ down to low $10^{-10}$ Torr as shown in Fig. 3. Since the insertion devices being installed in the straight sections may occupy $1 / 4 \sim 1 / 5$ of the circumference of the ring, the pressure reduction can be done in part by reducing the pressure inside the insertion device vacuum chambers.

In summary, we measured the beam-gas lifetime using helium gas and the vertical scraper in order to understand the beam-lifetime limiting process in a medium energy machine like the $2 \mathrm{GeV}$ PLS. It is found that for a medium to large gap vacuum chamber, the beam-gas scattering determines the beam lifetime at high pressures, $>5 \times 10^{-8}$ Torr. And the pressure independent Touschek effect mainly limits the lifetime and the contribution of the beam-gas lifetime to the total lifetime is small at the machine operation pressure of low $10^{-9}$ Torr. However, for a very narrow-gap undulator, Coulomb scattering may compete with the Touschek effect even at $\sim 1 \times 10^{-9}$ Torr indicating that the vacuum is still an important factor that determines the lifetime in a medium-energy, third generation light source.

\section{REFERENCES}

* Work supported by MOST and POSCO fund.

[1] J. Le Duff, Nucl. Instr. And Meth. A239, 83 (1985)

[2] Y. Miyahara and H. Nishimura, IEEE Trans. Nucl. Sci. NS-32, 3821 (1985).

[3] W. decking, et al, Presented at the Sixth European Particle Accelerator conference EPAC98, Stockholm, 1998.

[4] C. J. Bocchetta, et al, Presented at the Particle Accelerator conference PAC97, Vancouver, 1997.

[5] Annick Ropert, Presented at the Sixth European Particle Accelerator conference EPAC98, Stockholm, 1998. 